\title{
Stellar Shocks from Dark Matter Asteroid Impacts
}

\author{
Anirban Das $\odot,{ }^{1, *}$ Sebastian A. R. Ellis $\odot,{ }^{2,1, \dagger}$ Philip C. Schuster@, ${ }^{1, *}$ and Kevin Zhou $\odot^{1, \S}$ \\ ${ }^{1}$ SLAC National Accelerator Laboratory, 2575 Sand Hill Road, Menlo Park, California 94025, USA \\ ${ }^{2}$ Institut de Physique Théorique, Université Paris Saclay, CEA, F-91191 Gif-sur-Yvette, France
}

(Received 8 July 2021; revised 26 October 2021; accepted 15 December 2021; published 11 January 2022)

\begin{abstract}
Macroscopic dark matter is almost unconstrained over a wide "asteroidlike" mass range, where it could scatter on baryonic matter with geometric cross section. We show that when such an object travels through a star, it produces shock waves that reach the stellar surface, leading to a distinctive transient optical, UV, and x-ray emission. This signature can be searched for on a variety of stellar types and locations. In a dense globular cluster, such events occur far more often than flare backgrounds, and an existing UV telescope could probe orders of magnitude in dark matter mass in one week of dedicated observation.
\end{abstract}

DOI: 10.1103/PhysRevLett.128.021101

Astronomical and cosmological observations have provided all evidence for dark matter (DM) thus far. Stars, substellar objects, and stellar remnants therefore comprise natural venues for probing the nature of DM. The capture of particle DM is well-studied, and can result in signatures ranging from heating [1-4] and modifications of stellar structure $[5,6]$ to outright destruction by the formation of a black hole [7-9]. DM candidates light enough to be produced thermally in stars, such as the axion, can also be constrained by stellar cooling rates [10].

However, DM could also be in the form of objects of macroscopic mass and size, a possibility that is consistent with all cosmological constraints [11-13]. While macroscopic DM arises in many theoretical scenarios, it is difficult to detect terrestrially, primarily because such objects are rare given the low local DM density. As $M_{\mathrm{DM}}$ increases, experimental searches require either large detection volumes or long integration times. For example, for $M_{\mathrm{DM}} \lesssim 10^{5} \mathrm{~kg}$, limits on macroscopic DM passing near the Earth can be set with tabletop experiments, calorimeters, and gravitational wave detectors [14,15] or with searches for fast-moving meteors [16-18] and seismic waves $[19,20]$. However, for $M_{\mathrm{DM}} \gtrsim 10^{-20} M_{\odot}$, corresponding to a heavy asteroid, macroscopic DM would not have collided with Earth since the advent of human civilization, and setting constraints requires speculative appeals to geologic history [21,22]. Unambiguously probing the mass range $10^{-20} M_{\odot} \lesssim M_{\mathrm{DM}} \lesssim 10^{-11} M_{\odot}$ of "dark asteroids" will therefore require looking to the stars.

Published by the American Physical Society under the terms of the Creative Commons Attribution 4.0 International license. Further distribution of this work must maintain attribution to the author(s) and the published article's title, journal citation, and DOI. Funded by SCOAP ${ }^{3}$.
In this Letter, we point out that because dark asteroids move supersonically in stars, dissipation through any nongravitational interaction will generate shock waves. This allows the dissipated energy to quickly propagate to the stellar surface, where it is released in the form of a transient, thermal ultraviolet (UV) emission. Crucially, such events are correlated with the local DM density, but uncorrelated with the underlying activity of the star. Next-generation survey telescopes would detect such events without requiring a dedicated search, while existing telescopes could find them by monitoring regions of high DM density. This would constitute a DM direct detection experiment on astronomical scales, with the stars as the detector volume.

A detailed overview of models that produce dark asteroids is beyond the scope of this work, but the reader can keep several scenarios in mind. Self-interactions in the dark sector allow models as simple as asymmetric DM [23] to build up composite objects of high multiplicity in the early universe [24,25] and support compact structures [2628]. Additionally introducing a lighter, oppositely charged particle allows dark atoms to form, providing an alternative cooling mechanism that can generate large DM structures [29], while charging the DM under a non-Abelian gauge group naturally allows dark nucleosynthesis [30,31]. An even richer dark sector, which could result from mirroring part or all of the standard model (SM), allows the formation of mirror stars [32-35]. Phase transitions in the dark sector can also produce large dark objects from both bosonic and fermionic DM [36-39], with the density determined by the temperature of the phase transition.

For concreteness, we will introduce our signature by assuming that all DM is in the form of spherical dark asteroids with the same mass $M_{\mathrm{DM}}$ and radius $R_{\mathrm{DM}}$. We further assume that they scatter baryons elastically with geometric cross section $\sigma=\pi R_{\mathrm{DM}}^{2}$, enter the star head on, and do not disintegrate while passing through the star. 
In the final section, we discuss how these properties can arise and how the signature changes when they are relaxed.

Stellar collisions.-We compute stellar profiles with the Modules for Experiments in Stellar Astrophysics (MESA) code [40-44], assuming solar metallicity and the settings recommended by the MESA Isochrones and Stellar Tracks project [45], and match them at the photosphere to atmospheric profiles computed with PHOENIX [46]. When a dark asteroid enters a star of mass $M_{\star}$ and radius $R_{\star}$, it will be traveling at roughly the escape velocity $v_{\text {esc }}=\sqrt{2 G M_{\star} / R_{\star}}$, and is therefore hypersonic, with Mach number Ma $\sim 100$. It is accelerated inward by gravity and dissipates energy due to a drag force $\rho \sigma v^{2} c_{d} / 2$, where $c_{d} \simeq 1$ for a supersonic sphere [47]. For most of the parameters we consider, the dark asteroid remains hypersonic until it either dissipates most of its energy to drag or reaches the hot stellar core.

Describing the resulting production and propagation of shock waves is a complex hydrodynamic problem. However, it can be decomposed into simpler problems, each solvable by controlled approximations, as shown in more detail in the Supplemental Material [48]. First, because the dark asteroid is hypersonic, Ma $\gg 1$, its passage can be treated as an instantaneous deposition of energy $F_{\text {dr }}$ per unit length, which creates a cylindrical blast wave. Numeric blast wave solutions are known, and are used to model meteors traversing the Earth's atmosphere $[64,65]$. The shock wave becomes weak after it travels a characteristic radial distance $R_{0}=\sqrt{2 F_{\mathrm{dr}} / p} \sim \mathrm{Ma} R_{\mathrm{DM}}$, and asymptotically approaches an $N$-wave profile, a weak shock solution characterized by a pressure discontinuity $\Delta p$ and length $L$. Following Ref. [64], we match a blast wave onto an $N$-wave profile at distance $10 R_{0}$, where the shock strength is $\Delta p / p=0.06$, the length is $L=2.8 R_{0}$, and roughly half of the original energy remains in the shock wave.

To treat the propagation to the stellar surface, we use standard results from weak shock theory $[66,67]$. In particular, the propagation of a weak shock wave through a slowly varying medium can be described by geometric acoustics. Because the speed of sound decreases with distance from the center of the star, the ray paths refract radially outward. We propagate each piece of the shock front along such a ray. For an acoustic wave, if the wavefront area evolves as $A(s)$ along a ray, then the pressure amplitude varies as $\Delta p \propto \sqrt{\rho c_{s} / A(s)}$, while the period $L / c_{s}$ remains constant. The discontinuities of an $N$ wave cause additional dissipation: when the shock wave travels a length $L$, there is a fractional increase in $L$ and a fractional decrease in shock strength and total energy of order $\Delta p / p$.

Finally, as each piece of the shock front approaches the stellar surface, the decreasing density and pressure cause a rapid increase in the shock strength. For the DM masses and radii of interest here, the shock becomes strong, $\Delta p / p \gtrsim 1$, below the photosphere, at optical depths up to $\sim 10^{2}$. Analytic solutions exist to describe the arrival of a strong shock wave at the edge of a star [68]. To roughly approximate these results, we assume that once the shock wave becomes strong, its remaining energy heats the stellar material above it to a uniform temperature $T_{f}$, which sets the typical frequency band of emission. This is reasonable because convection in the shocked region near the stellar surface will effectively smooth out temperature gradients. The timescale for energy release is then dictated by the rate of blackbody radiation from the surface and is typically on the order of hundreds of seconds.

This treatment is compatible with previous work on shock waves in stars. In massive stars just prior to core collapse, convection can excite acoustic waves [69], which then steepen into weak shocks that dissipate in the same way as they travel outward [70,71]. Refs. [72,73] considered the strengthening of a shock wave near the surface of a star; consistent with this work, we find that our shocks are insufficiently energetic to eject mass, as they emerge with a typical speed $\sqrt{k_{B} T_{f} / m_{p}} \ll v_{\text {esc }}$.

In Fig. 1, we show the total shock energy released from the surface of a Sun-like star and the typical final temperature $T_{f}$. The qualitative features of this plot can be readily understood. For higher $R_{\mathrm{DM}}$, the dark asteroid stops near the stellar surface, and a small portion of the surface is heated to a high temperature. As $R_{\mathrm{DM}}$ decreases, the shock waves are primarily produced deeper in the star, with a shorter wavelength. This increases the dissipation they experience as they propagate out to the surface, decreasing the energy released. At the smallest radii, drag is insufficient to prevent the dark asteroid from passing through the

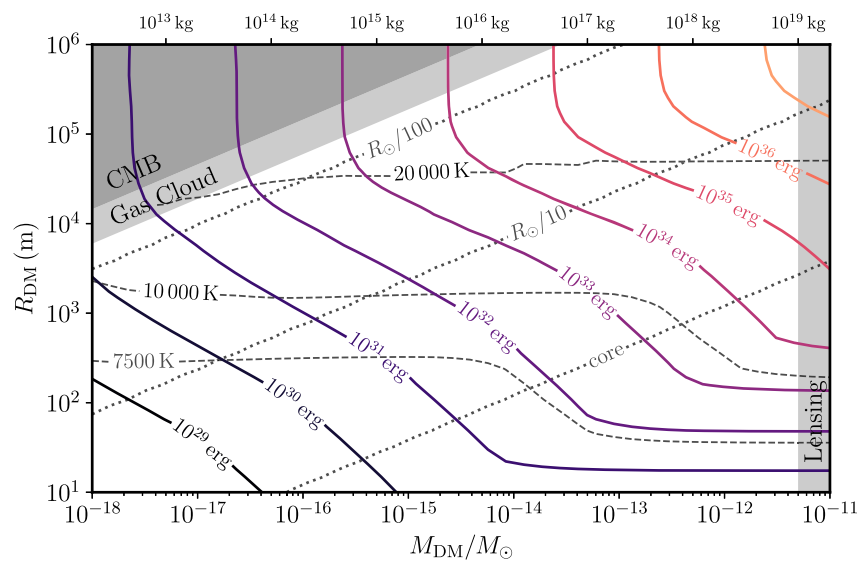

FIG. 1. Contours of energy release (solid), characteristic temperature (dashed), and penetration depth (dotted) for a dark asteroid impact on a Sun-like star. We show bounds from the cosmic microwave background limit on DM-baryon scattering [74], heating of cold gas clouds [75] (though see also Ref. [76]), and microlensing [77] (though see also Refs. [78-80]). We do not show constraints from femtolensing of gamma-ray bursts [81], which are weakened by finite source size effects [82]. 
entire star, so that only part of its energy is deposited, leading to a rapid falloff in signal energy.

Since a strong shock has $\Delta T / T \sim 1$, the temperature $T_{f}$ roughly tracks the local temperature at the depth where the weak shock becomes strong again; as a result, it is relatively insensitive to $M_{\mathrm{DM}}$ and $R_{\mathrm{DM}}$ and typically peaks in the far UV. At lower densities, $T_{f}$ rapidly rises because the dark asteroid stops so close to the surface that the shock never becomes weak. At the very lowest densities shown, the dark asteroid stops above the photosphere. In this extreme case, the emission spectrum is not necessarily thermal and depends on the detailed physics of the resulting plasma. We do not study this regime because it is in tension with cosmological constraints, but we expect photons to be released at up to x-ray energies, $\epsilon \sim m_{p} v_{\mathrm{esc}}^{2} \sim 10^{4} \mathrm{eV}$.

Similar results apply to other star types and are shown in the Supplemental Material [48]. The main difference is that for equal $M_{\mathrm{DM}}$ and $R_{\mathrm{DM}}$, the signal energy is higher for more compact objects, such as red and brown dwarfs, because their density profiles rise more steeply with depth, causing the dark asteroid's energy to be deposited closer to the surface. Conversely, the signal energy is significantly lower for giant stars because of their extended envelopes.

Observational prospects.-Dark asteroids are expected to produce rare transients on all types of stars, with a frequency dependent on the stellar and local DM parameters. For a star moving with a DM halo, averaging over a Maxwellian velocity distribution for the DM yields the collision rate [83]

$$
\Gamma=\sqrt{\frac{8}{3 \pi}} \frac{\rho_{\mathrm{DM}} v_{d}}{M_{\mathrm{DM}}} \pi R_{\star}^{2}\left(1+\frac{3 v_{\mathrm{esc}}^{2}}{2 v_{d}^{2}}\right),
$$

where $v_{d}$ is the velocity dispersion. The final term accounts for the focusing effect of gravitational attraction. In all cases we will consider, $v_{\mathrm{esc}} \gg v_{d}$, giving

$$
\begin{aligned}
\Gamma \simeq & \left(4 \times 10^{-5} \mathrm{yr}^{-1}\right) \frac{M_{\star}}{M_{\odot}} \frac{R_{\star}}{R_{\odot}} \\
& \times \frac{10^{-15} M_{\odot}}{M_{\mathrm{DM}}} \frac{\rho_{\mathrm{DM}}}{0.4 \mathrm{GeV} / \mathrm{cm}^{3}} \frac{270 \mathrm{~km} / \mathrm{s}}{v_{d}} .
\end{aligned}
$$

As shown in the inset of Fig. 2, we expect a brief x-ray emission as the dark asteroid passes through the stellar atmosphere, followed by a gradual optical and UV emission as the shock wave produced inside reaches the surface of the star. Since most of the energy emerges in the UV and cooler stars emit relatively little in this band, it is easiest to search for these events as UV transients.

The light curve would also have a long tail as the violently heated patch of the stellar surface gradually cools, which could be targeted for follow-up optical observation. Note that we have treated all collisions as head on, though the high degree of gravitational focusing implies that most

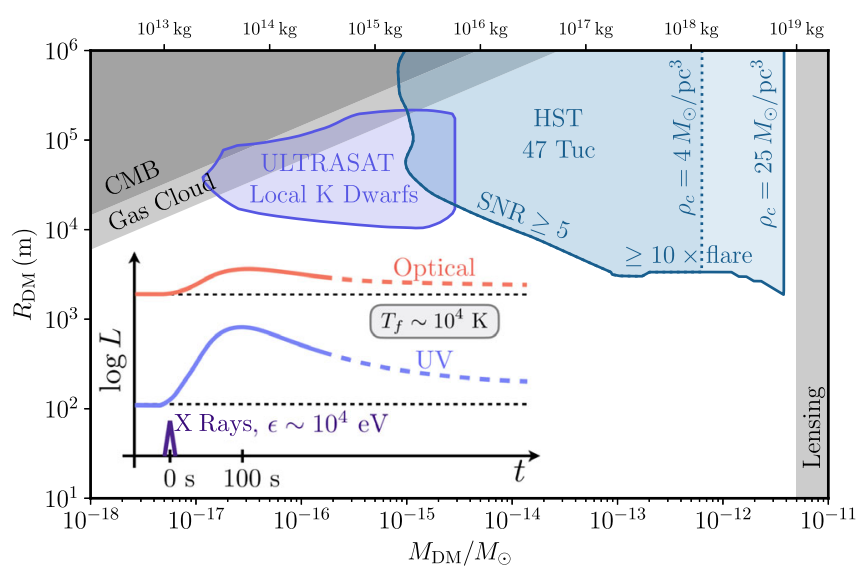

FIG. 2. Contour plot showing observability. We shade regions where impacts on $\mathrm{K}$ dwarfs within $1 \mathrm{kpc}$ would be seen by ULTRASAT, and impacts on Sun-like stars in 47 Tuc would be seen by the Hubble Space Telescope, at least once per year and week of observation on average, respectively. In both cases, we demand SNR $\geq 5$. For 47 Tuc, we show two possible values of the core DM density, as discussed in the main text, and require the rate of dark asteroid impacts to exceed superflares of similar energy by at least an order of magnitude. A schematic light curve for three frequency bands is shown in the inset.

collisions are glancing. Our calculation is thus maximally conservative because it gives the shock waves the longest possible path to the surface. Accounting for the impact parameters would increase the signal strength and temperature and could also increase visibility to x-ray telescopes.

Upcoming transient surveys could detect dark asteroid collisions on nearby stars without requiring a dedicated search. Among star types, K dwarfs are promising targets as they have significantly larger masses and radii than $\mathrm{M}$ dwarfs but also have a higher number density and negligible UV emission compared to hotter stars. As a concrete example, we consider ULTRASAT [84], a proposed widefield UV transient explorer designed to detect distant supernova shock breakouts, which will also monitor many nearby stars. We compute the maximum distance from which ULTRASAT could observe dark asteroid collisions at $\mathrm{SNR} \geq 5$, conservatively counting only impacts on $\mathrm{K}$ dwarfs, and approximate the star density as uniform out to $1 \mathrm{kpc}$ from the Earth. The observable region of parameter space is cut off at high $R_{\mathrm{DM}}$ because the signal temperature becomes too high, at low $R_{\mathrm{DM}}$ and $M_{\mathrm{DM}}$ because the signal energy becomes too low, and at high $M_{\mathrm{DM}}$ because the events become too rare.

A similar region could be probed by the upcoming Legacy Survey of Space and Time (LSST) [85], but estimating the event rate is more difficult because of the LSST's complex observing strategy and multiple filters. In addition, since the LSST would be able to see events at a significantly larger distance $d \gtrsim \mathrm{kpc}$, a more detailed model of the galactic stellar and DM densities would be required, along with estimates of UV extinction. Exoplanet searches 
such as those by the Transiting Exoplanets Survey Satellite [86] and the planned PLATO mission [87] have exceptionally large fields of view but observe in the red, which reduces the sensitivity because of stellar variability and shot noise. However, these instruments could effectively detect transients on cool red dwarfs or brown dwarfs, which thereby probes lower $R_{\mathrm{DM}}$, as shown in the Supplemental Material [48].

Because the local DM density is low, a potential obstacle for any local search is the background from stellar superflares, which occupy a similar temperature and energy range. Observations from Kepler $[88,89]$ and the Transiting Exoplanets Survey Satellite [90] find no superflares on the vast majority of FGK dwarfs and almost none on those that are not rotating rapidly, which allows highly active stars to be excluded from observation. However, for any individual event, it would be difficult to rule out the possibility of a superflare without further information. For example, follow-up observations could determine the detailed light curve, which could fall off more slowly for dark asteroid impacts because the energy emerges from within the star rather than from its atmosphere. Simultaneous observation with other instruments could rule out flares using spectral information, as they are expected to have a significant radio and $\mathrm{x}$-ray component.

An alternative strategy is to perform a focused search in a region where the impact rate per star is significantly higher. As a concrete example, we consider 47 Tuc (NGC 104), a well-studied nearby $(d \sim 4 \mathrm{kpc})$ globular cluster that has a dense core and negligible UV dust extinction [91].

While the DM content of globular clusters today is not known $[92,93]$, they are thought to have formed in large DM subhalos [94,95], with computational studies suggesting an initial DM mass of about 260 times the stellar mass [96]. Tidal stripping and DM thermalization are expected to have reduced the DM content of the globular cluster since formation [97], with $\sim 1 \%$ remaining today [98]. We assume this formation history holds for 47 Tuc, and model the DM distribution with a Navarro-FrenkWhite profile [99]. Gravitational interactions transfer kinetic energy to the dark asteroids and lighter stars, which we account for by coring the DM profile [100] and taking a relatively heavy benchmark star of solar mass.

From the above procedure, detailed in the Supplemental Material [48], we infer a core DM density $\rho_{\mathrm{DM}} \simeq 4 M_{\odot} / \mathrm{pc}^{3}$. Since the velocity dispersion is $v_{d} \simeq 12 \mathrm{~km} / \mathrm{s}$, the collision rate per star is almost 4 orders of magnitude higher than in the local region, even though $\mathrm{DM}$ is still a vastly subdominant component of the core. For most of the parameters we consider, the event rate exceeds the rate of superflares of comparable energy on Sun-like stars [89] by orders of magnitude. Yet our estimate is conservative, as a recent analysis with similar assumptions [1] found a core DM density $\rho_{\mathrm{DM}} \simeq 25 M_{\odot} / \mathrm{pc}^{3}$ [101]. Furthermore, we neglect adiabatic contraction of the DM halo [102,103], which would significantly increase the core density, and we do not consider the possibility of a DM cusp due to an intermediate-mass black hole [104,105], within which the DM density would be enhanced by orders of magnitude.

To monitor 47 Tuc, we consider the Wide Field Camera 3 instrument on the Hubble Space Telescope, using the F225W filter. This instrument's field of view is sufficient to capture most of the DM core, and the UV filter alleviates stellar crowding [106]. In Fig. 2, we show the region where at least one event with SNR $\geq 5$ is expected in one week of continuous observation. The Hubble telescope has in fact already monitored 47 Tuc for over a week to search for exoplanets [107], though these optical and infrared observations are less useful for our purposes due to stellar backgrounds. Since the event rate scales as $1 / M_{\mathrm{DM}}$, new parameter space could be probed with as little as one hour of dedicated UV observation.

Discussion.-For concreteness, we have focused on specific assumptions and experimental searches, but our results also apply more generally. For instance, we have taken elastic scattering as a generic benchmark, but specific models can give rise to nonelastic interactions such as catalyzing proton decay, annihilating with ordinary matter, or absorbing part of the dissipated energy. We have also assumed a geometric cross section for baryon scattering because it is the result of any sufficiently strong interaction that is not long-ranged, but the dark asteroid can be partly transparent to baryons, or interact by a long-range force, yielding a smaller or larger cross section, respectively. These effects can be accounted for by simply scaling the energy deposited per length $F_{\mathrm{dr}}$, as long as $R_{0} \gtrsim R_{\mathrm{DM}}$.

The assumption of geometric cross section implies relatively strong DM interactions with the SM, and it is interesting to see how this can be compatible with existing constraints. As shown in Fig. 1, cosmological constraints are relatively weak, essentially because dark asteroids would be extremely rare, and the constraint from DM self-interaction in the Bullet cluster is orders of magnitude weaker. Strong DM constituent interactions with the SM could be accommodated if, for example, the dark asteroid was composed of "dark atoms" of oppositely charged particles bound by a dark $U(1)$. If the constituent has $m_{\mathrm{DM}} \lesssim 100 \mathrm{MeV}$ and less than $1 \%$ is unbound, terrestrial constraints require that the DM-nucleon cross section not exceed $\sigma_{\mathrm{SI}} \lesssim 10^{-29} \mathrm{~cm}^{2}[108,109]$. A dark asteroid of density $\mathrm{g} / \mathrm{cm}^{3}$ containing $100 \mathrm{MeV}$ constituents with this SM interaction strength would have a mean free path of $\sim 100 \mathrm{~m}$, so that the assumption of opacity holds for the regions of interest of Fig. 2.

Our rough estimates of the collision rate and signal energy could be refined in many ways. We have taken all dark asteroids to have the same mass $M_{\mathrm{DM}}$, though a realistic production mechanism would lead to a range of masses. This would not necessarily harm prospects for a 
local search, as impacts of heavier dark asteroids could be seen from further away. Dark asteroids could pass through a star but lose sufficient energy to be captured, ensuring subsequent collisions. This enhancement of the event rate occurs for a wide range of $M_{\mathrm{DM}}$, because most collisions are glancing, and leads to the intriguing possibility of follow-up detection. Finally, our treatment of the shock propagation and convective energy transport near the surface could be improved with detailed analytic arguments or numerics, which would also yield the detailed shape of the light curve.

Collision events have been investigated in related contexts, though the results are qualitatively different. Primordial black holes passing through stars deposit a small amount of energy through dynamical friction, but the result is too weak to observe [110,111]. Dark asteroids could also trigger supernovae in white dwarfs by depositing energy in their interiors $[112,113]$. The survival of white dwarfs therefore implies a strong constraint on macroscopic DM due to the long effective integration time, but it only applies to dark asteroids of roughly nuclear density, which can penetrate the white dwarf's crust.

Within the standard model, the closest analogue to a dark asteroid impact would be a comet impact [114]. However, comets are expected to be rare outside of planetary systems, with the interstellar comet density bounded orders of magnitude below the DM density [115]. Comets are also "rubble piles" that fall apart before even reaching the stellar surface, leading to a qualitatively different signature. By contrast, in simple dark sector models, the binding energy of a dark asteroid may easily exceed its kinetic energy, which is only about $\left(v_{d} / c\right)^{2} \sim 10^{-6}$ of its total mass energy, implying that ablation is a small effect.

Many additional directions could be explored in future work. For instance, the high DM density at the galactic center would make it ideal for a focused search, though one would have to model its distinct stellar populations and use a sightline with low extinction. Globular clusters besides 47 Tuc could be promising, especially if new nearby clusters are found, or confirmed to contain an intermediate-mass black hole. Milky Way satellite galaxies are more distant but are known to host a high DM density, and could likely be used to probe higher $M_{\mathrm{DM}}$. At the opposite end of the mass range, impacts on the Sun are expected to occur annually for $M_{\mathrm{DM}} \lesssim 10^{-19} M_{\odot}$ and would be energetic enough to be easily detected by solar observatories. It would be interesting to see if the resolution of these instruments permits such impacts to be distinguished from solar flares. In many of these cases, it may be possible to find impact events in a reanalysis of archival data.

The possibility of detecting dark asteroid impacts in nearby stars provides an interesting target for UV transient searches with small satellites [116-118], while more powerful instruments would be well-suited for focused searches. These observations are enabled by the rapid advance of time-domain astronomy, which we have shown provides an unusual route to discovering the nature of dark matter.

We thank Patrick Eggenberger, Rebecca Leane, Bruce Macintosh, Eric Mamajek, Georges Meynet, Payel Mukhopadhyay, Stephen Ro, Ningqiang Song, and Natalia Toro for helpful discussions. A. D., S. A. R. E., P. S., and K.Z. were supported by the U.S. Department of Energy under Contract No. DE-AC02-76SF00515 while at SLAC. S. A. R. E. was also partially supported by Schweizerischer Nationalfonds grant P400P2_186678. K. Z. is supported by the NSF GRFP under grant DGE-1656518.

* Corresponding author.

anirband@slac.stanford.edu

Corresponding author.

sarellis@protonmail.com

Corresponding author.

schuster@slac.stanford.edu

${ }^{\S}$ Corresponding author.

knzhou@stanford.edu

[1] G. Bertone and M. Fairbairn, Compact stars as dark matter probes, Phys. Rev. D 77, 043515 (2008).

[2] C. Kouvaris, WIMP annihilation and cooling of neutron stars, Phys. Rev. D 77, 023006 (2008).

[3] M. Baryakhtar, J. Bramante, S. W. Li, T. Linden, and N. Raj, Dark Kinetic Heating of Neutron Stars and an Infrared Window On WIMPs, SIMPs, and Pure Higgsinos, Phys. Rev. Lett. 119, 131801 (2017).

[4] R. K. Leane and J. Smirnov, Exoplanets as Sub-GeV Dark Matter Detectors, Phys. Rev. Lett. 126, 161101 (2021).

[5] F. Iocco, M. Taoso, F. Leclercq, and G. Meynet, Main Sequence Stars with Asymmetric Dark Matter, Phys. Rev. Lett. 108, 061301 (2012).

[6] J. Casanellas and I. Lopes, First asteroseismic limits on the nature of dark matter, Astrophys. J. Lett. 765, L21 (2013).

[7] I. Goldman and S. Nussinov, Weakly interacting massive particles and neutron stars, Phys. Rev. D 40, 3221 (1989).

[8] J. F. Acevedo, J. Bramante, A. Goodman, J. Kopp, and T. Opferkuch, Dark matter, destroyer of worlds: neutrino, thermal, and existential signatures from black holes in the sun and earth, J. Cosmol. Astropart. Phys. 04 (2021) 026.

[9] B. Dasgupta, R. Laha, and A. Ray, Low Mass Black Holes from Dark Core Collapse, Phys. Rev. Lett. 126, 141105 (2021).

[10] G. G. Raffelt, Stars as Laboratories for Fundamental Physics: The Astrophysics of Neutrinos, Axions, and Other Weakly Interacting Particles (University of Chicago Press, Chicago, 1996).

[11] D. M. Jacobs, G. D. Starkman, and B. W. Lynn, Macro dark matter, Mon. Not. R. Astron. Soc. 450, 3418 (2015).

[12] S. Burdin, M. Fairbairn, P. Mermod, D. Milstead, J. Pinfold, T. Sloan, and W. Taylor, Non-collider searches for stable massive particles, Phys. Rep. 582, 1 (2015). 
[13] Y. Bai, A. J. Long, and S. Lu, Tests of Dark MACHOs: lensing, accretion, and glow, J. Cosmol. Astropart. Phys. 09 (2020) 044.

[14] D. M. Grabowska, T. Melia, and S. Rajendran, Detecting dark blobs, Phys. Rev. D 98, 115020 (2018).

[15] D. Budker, V. V. Flambaum, X. Liang, and A. Zhitnitsky, Axion quark nuggets and how a global network can discover them, Phys. Rev. D 101, 043012 (2020).

[16] J. Singh Sidhu, R. M. Abraham, C. Covault, and G. Starkman, Macro detection using fluorescence detectors, J. Cosmol. Astropart. Phys. 02 (2019) 037.

[17] J. S. Sidhu and G. Starkman, Macroscopic dark matter constraints from bolide camera networks, Phys. Rev. D 100, 123008 (2019).

[18] L. W. Piotrowski, K. Małek, L. Mankiewicz, M. Sokołowski, G. Wrochna, A. Zadrożny, and A. F. Żarnecki, Limits on the Flux of Nuclearites and Other Heavy Compact Objects from the Pi of the Sky Project, Phys. Rev. Lett. 125, 091101 (2020).

[19] E. T. Herrin, D. C. Rosenbaum, and V. L. Teplitz, Seismic search for strange quark nuggets, Phys. Rev. D 73, 043511 (2006).

[20] D. Cyncynates, J. Chiel, J. Sidhu, and G. D. Starkman, Reconsidering seismological constraints on the available parameter space of macroscopic dark matter, Phys. Rev. D 95, 063006 (2017); 95, 129903(A) (2017).

[21] S. Abbas and A. Abbas, Volcanogenic dark matter and mass extinctions, Astropart. Phys. 8, 317 (1998).

[22] J. Rafelski, L. Labun, and J. Birrell, Compact Ultradense Matter Impactors, Phys. Rev. Lett. 110, 111102 (2013).

[23] K. M. Zurek, Asymmetric dark matter: Theories, signatures, and constraints, Phys. Rep. 537, 91 (2014).

[24] E. Hardy, R. Lasenby, J. March-Russell, and S. M. West, Big bang synthesis of nuclear dark matter, J. High Energy Phys. 06 (2015) 011.

[25] M. I. Gresham, H. K. Lou, and K. M. Zurek, Early Universe synthesis of asymmetric dark matter nuggets, Phys. Rev. D 97, 036003 (2018).

[26] C. Kouvaris and N. G. Nielsen, Asymmetric dark matter stars, Phys. Rev. D 92, 063526 (2015).

[27] M. I. Gresham, H. K. Lou, and K. M. Zurek, Astrophysical signatures of asymmetric dark matter bound states, Phys. Rev. D 98, 096001 (2018).

[28] J. H. Chang, D. Egana-Ugrinovic, R. Essig, and C. Kouvaris, Structure formation and exotic compact objects in a dissipative dark sector, J. Cosmol. Astropart. Phys. 03 (2019) 036.

[29] M. R. Buckley and A. DiFranzo, Collapsed Dark Matter Structures, Phys. Rev. Lett. 120, 051102 (2018).

[30] G. Krnjaic and K. Sigurdson, Big bang darkleosynthesis, Phys. Lett. B 751, 464 (2015).

[31] W. Detmold, M. McCullough, and A. Pochinsky, Dark nuclei I: Cosmology and indirect detection, Phys. Rev. D 90, 115013 (2014).

[32] R. Foot, Mirror matter-type dark matter, Int. J. Mod. Phys. D 13, 2161 (2004).

[33] Z. Berezhiani, S. Cassisi, P. Ciarcelluti, and A. Pietrinferni, Evolutionary and structural properties of mirror star MACHOs, Astropart. Phys. 24, 495 (2006).
[34] G. D’Amico, P. Panci, A. Lupi, S. Bovino, and J. Silk, Massive black holes from dissipative dark matter, Mon. Not. R. Astron. Soc. 473, 328 (2018).

[35] D. Curtin and J. Setford, Signatures of mirror stars, J. High Energy Phys. 03 (2020) 041.

[36] E. Witten, Cosmic separation of phases, Phys. Rev. D 30, 272 (1984).

[37] J. A. Frieman, G. B. Gelmini, M. Gleiser, and E. W. Kolb, Solitogenesis: Primordial Origin of Nontopological Solitons, Phys. Rev. Lett. 60, 2101 (1988).

[38] Y. Bai, A. J. Long, and S. Lu, Dark quark nuggets, Phys. Rev. D 99, 055047 (2019).

[39] C. Gross, G. Landini, A. Strumia, and D. Teresi, Dark matter as dark dwarfs and other macroscopic objects: multiverse relics? J. High Energy Phys. 09 (2021) 033.

[40] B. Paxton, L. Bildsten, A. Dotter, F. Herwig, P. Lesaffre, and F. Timmes, Modules for experiments in stellar astrophysics (MESA), Astrophys. J. Suppl. Ser. 192, 3 (2011).

[41] B. Paxton, M. Cantiello, P. Arras, L. Bildsten, E. F. Brown, A. Dotter, C. Mankovich, M. H. Montgomery, D. Stello, F. X. Timmes, and R. Townsend, Modules for experiments in stellar astrophysics (MESA): Planets, oscillations, rotation, and massive stars, Astrophys. J. Suppl. Ser. 208, 4 (2013).

[42] B. Paxton, P. Marchant, J. Schwab, E. B. Bauer, L. Bildsten, M. Cantiello, L. Dessart, R. Farmer, H. Hu, N. Langer, R. H. D. Townsend, D. M. Townsley, and F. X. Timmes, Modules for experiments in stellar astrophysics (MESA): Binaries, pulsations, and explosions, Astrophys. J. Suppl. Ser. 220, 15 (2015).

[43] B. Paxton, J. Schwab, E. B. Bauer, L. Bildsten, S. Blinnikov, P. Duffell, R. Farmer, J. A. Goldberg, P. Marchant, E. Sorokina, A. Thoul, R. H. D. Townsend, and F.X. Timmes, Modules for experiments in stellar astrophysics (MESA): Convective boundaries, element diffusion, and massive star explosions, Astrophys. J. Suppl. Ser. 234, 34 (2018).

[44] B. Paxton, R. Smolec, J. Schwab, A. Gautschy, L. Bildsten, M. Cantiello, A. Dotter, R. Farmer, J. A. Goldberg, A. S. Jermyn, S. M. Kanbur, P. Marchant, A. Thoul, R. H. D. Townsend, W. M. Wolf, M. Zhang, and F. X. Timmes, Modules for experiments in stellar astrophysics (MESA): Pulsating variable stars, rotation, convective boundaries, and energy conservation, Astrophys. J. Suppl. Ser. 243, 10 (2019).

[45] J. Choi, A. Dotter, C. Conroy, M. Cantiello, B. Paxton, and B. D. Johnson, MESA isochrones and stellar tracks (MIST). I. Solar-scaled models, Astrophys. J. 823, 102 (2016).

[46] T. O. Husser, S. Wende-von Berg, S. Dreizler, D. Homeier, A. Reiners, T. Barman, and P. H. Hauschildt, A new extensive library of PHOENIX stellar atmospheres and synthetic spectra, Astron. Astrophys. 553, A6 (2013).

[47] A. Bailey and J. Hiatt, Sphere drag coefficients for a broad range of Mach and Reynolds numbers, AIAA J. 10, 1436 (1972).

[48] See Supplemental Material at http://link.aps.org/ supplemental/10.1103/PhysRevLett.128.021101 for further details on the shock propagation, results for brown dwarfs and red giants, the stellar and dark matter profiles of 47 Tuc, and estimates of telescope sensitivity, which includes Refs. [49-63]. 
[49] M. N. Plooster, Shock waves from line sources. Numerical solutions and experimental measurements, Phys. Fluids 13, 2665 (1970).

[50] K. Zahnle and M.-M. Mac Low, The collision of Jupiter and comet Shoemaker-Levy 9, Icarus 108, 1 (1994).

[51] A. Anic, Y. Alibert, and W. Benz, Giant collisions involving young Jupiter, Astron. Astrophys. 466, 717 (2007).

[52] H. Baumgardt, M. Hilker, A. Sollima, and A. Bellini, Mean proper motions, space orbits, and velocity dispersion profiles of Galactic globular clusters derived from Gaia DR2 data, Mon. Not. R. Astron. Soc. 482, 5138 (2019).

[53] I. King, The structure of star clusters. I. An empirical density law, Astron. J. 67, 471 (1962).

[54] F. Campos, P. Bergeron, A. D. Romero, S. O. Kepler, G. Ourique, J. E. S. Costa, C. J. Bonatto, D. E. Winget, M. H. Montgomery, T. A. Pacheco, and L. R. Bedin, A comparative analysis of the observed white dwarf cooling sequence from globular clusters, Mon. Not. R. Astron. Soc. 456, 3729 (2016).

[55] K. Brogaard, D. A. VandenBerg, L. R. Bedin, A. P. Milone, A. Thygesen, and F. Grundahl, The age of 47 Tuc from self-consistent isochrone fits to colour-magnitude diagrams and the eclipsing member V69, Mon. Not. R. Astron. Soc. 468, 645 (2017).

[56] N. Aghanim, Y. Akrami, M. Ashdown, J. Aumont, C. Baccigalupi, M. Ballardini, A. J. Banday, R. B. Barreiro, N. Bartolo et al., Planck 2018 results, Astron. Astrophys. 641, A6 (2020).

[57] A. D. Ludlow, S. Bose, R. E. Angulo, L. Wang, W. A. Hellwing, J. F. Navarro, S. Cole, and C. S. Frenk, The mass-concentration-redshift relation of cold and warm dark matter haloes, Mon. Not. R. Astron. Soc. 460, 1214 (2016).

[58] B. Diemer and M. Joyce, An accurate physical model for halo concentrations, Astrophys. J. 871, 168 (2019).

[59] J. Wang, S. Bose, C. S. Frenk, L. Gao, A. Jenkins, V. Springel, and S. D. M. White, Universal structure of dark matter haloes over a mass range of 20 orders of magnitude, Nature (London) 585, 39 (2020).

[60] L. Spitzer, Dynamical Evolution of Globular Clusters (Princeton University Press, Princeton, 1987).

[61] E. Mamajek, Number densities of stars of different types in the solar vicinity, https://www.pas.rochester.edu/\%7eemamajek/ memo_star_dens.html (2021). Accessed: 2021-05-14.

[62] E. Waxman, ULTRASAT: A Wide-Field UV Space Telescope, https://www.weizmann.ac.il/ultrasat/sites/ultrasat/ files/uploads/1_Eli_2020\%2006\%20ULTRASAT\% 2015\%20\%2B\%205.pdf (2020), Accessed: 2021-05-14.

[63] R. A. Windhorst et al., The Hubble space telescope wide field camera 3 early release science data: Panchromatic faint object counts for $0.2-2 \mu \mathrm{m}$ wavelength, Astrophys. J. Suppl. Ser. 193, 27 (2011).

[64] D. O. ReVelle, On meteor-generated infrasound, J. Geophys. Res. 81, 1217 (1976).

[65] E. A. Silber and P. G. Brown, Optical observations of meteors generating infrasound-I: Acoustic signal identification and phenomenology, J. Atmos. Sol. Terr. Phys. 119, 116 (2014).

[66] G. Whitham, On the propagation of weak shock waves, J. Fluid Mech. 1, 290 (1956).
[67] A. D. Pierce, Acoustics: An Introduction to its Physical Principles and Applications (Springer, New York, 2019).

[68] A. Sakurai, On the problem of a shock wave arriving at the edge of a gas, Communs. Pure Appl. Math. 13, 353 (1960).

[69] E. Quataert and J. Shiode, Wave-driven mass loss in the last year of stellar evolution: setting the stage for the most luminous core-collapse supernovae, Mon. Not. R. Astron. Soc. 423, L92 (2012).

[70] S. Ro and C.D. Matzner, Shock dynamics in stellar outbursts: I. Shock formation, Astrophys. J. 841, 9 (2017).

[71] J. Fuller and S. Ro, Pre-supernova outbursts via wave heating in massive stars-II. Hydrogen-poor stars, Mon. Not. R. Astron. Soc. 476, 1853 (2018).

[72] C. D. Matzner and S. Ro, Wave-driven shocks in stellar outbursts: Dynamics, envelope heating, and nascent blast waves, Astrophys. J. 908, 23 (2021).

[73] I. Linial, J. Fuller, and R. Sari, Partial stellar explosionsejected mass and minimal energy, Mon. Not. R. Astron. Soc. 501, 4266 (2021).

[74] C. Dvorkin, K. Blum, and M. Kamionkowski, Constraining dark matter-baryon scattering with linear cosmology, Phys. Rev. D 89, 023519 (2014).

[75] A. Bhoonah, J. Bramante, S. Schon, and N. Song, Detecting composite dark matter with long range and contact interactions in gas clouds, Phys. Rev. D 103, 123026 (2021).

[76] D. Wadekar and G. R. Farrar, First astrophysical constraints on dark matter interactions with ordinary matter at low relative velocity, Phys. Rev. D 103, 123028 (2021).

[77] H. Niikura, M. Takada, N. Yasuda, R. H. Lupton, T. Sumi, S. More, T. Kurita, S. Sugiyama, A. More, M. Oguri, and M. Chiba, Microlensing constraints on primordial black holes with Subaru/HSC Andromeda observations, Nat. Astron. 3, 524 (2019).

[78] P. Montero-Camacho, X. Fang, G. Vasquez, M. Silva, and C. M. Hirata, Revisiting constraints on asteroid-mass primordial black holes as dark matter candidates, J. Cosmol. Astropart. Phys. 08 (2019) 031.

[79] N. Smyth, S. Profumo, S. English, T. Jeltema, K. McKinnon, and P. Guhathakurta, Updated constraints on asteroid-mass primordial black holes as dark matter, Phys. Rev. D 101, 063005 (2020).

[80] D. Croon, D. McKeen, N. Raj, and Z. Wang, Subaru-HSC through a different lens: Microlensing by extended dark matter structures, Phys. Rev. D 102, 083021 (2020).

[81] A. Barnacka, J. F. Glicenstein, and R. Moderski, New constraints on primordial black holes abundance from femtolensing of gamma-ray bursts, Phys. Rev. D 86, 043001 (2012).

[82] A. Katz, J. Kopp, S. Sibiryakov, and W. Xue, Femtolensing by dark matter revisited, J. Cosmol. Astropart. Phys. 12 (2018) 005.

[83] A. Gould, Resonant enhancements in weakly interacting massive particle capture by the earth, Astrophys. J. 321, 571 (1987).

[84] I. Sagiv, A. Gal-Yam, E. O. Ofek, E. Waxman, O. Aharonson, S. R. Kulkarni, E. Nakar, D. Maoz, B. Trakhtenbrot, E. S. Phinney, J. Topaz, C. Beichman, J. Murthy, and S. P. Worden, 
Science with a wide-field UV transient explorer, Astron. J. 147, 79 (2014).

[85] Ž. Ivezić, S. M. Kahn, J. A. Tyson, B. Abel, E. Acosta, R. Allsman, D. Alonso, Y. AlSayyad, S. F. Anderson, J. Andrew, J. R. P. Angel, G. Z. Angeli, R. Ansari, P. Antilogus, C. Araujo, R. Armstrong, K. T. Arndt et al., LSST: From science drivers to reference design and anticipated data products, Astrophys. J. 873, 111 (2019).

[86] G. R. Ricker, J. N. Winn, R. Vanderspek, D. W. Latham, G. Á. Bakos, J. L. Bean, Z. K. Berta-Thompson, T. M. Brown, L. Buchhave, N. R. Butler, R. P. Butler, W. J. Chaplin, D. Charbonneau, J. Christensen-Dalsgaard, M. Clampin et al., Transiting exoplanet survey satellite (TESS), in Space Telescopes and Instrumentation 2014: Optical, Infrared, and Millimeter Wave, edited by J. Oschmann, Jacobus M., M. Clampin, G. G. Fazio, and H. A. MacEwen, Vol. 9143 of Society of Photo-Optical Instrumentation Engineers (SPIE) Conference Series (SPIE, Bellingham, 2014), p. 914320, 10.1117/12.2063489.

[87] H. Rauer et al., The PLATO 2.0 mission, Exp. Astron. 38, 249 (2014).

[88] J. R. A. Davenport, The Kepler catalog of stellar flares, Astrophys. J. 829, 23 (2016).

[89] S. Okamoto, Y. Notsu, H. Maehara, K. Namekata, S. Honda, K. Ikuta, D. Nogami, and K. Shibata, Statistical properties of superflares on solar-type stars: results using all of the Kepler primary mission data, Astrophys. J. 906, 72 (2021).

[90] M. N. Günther et al., Stellar flares from the first TESS data release: Exploring a new sample of $\mathrm{M}$ dwarfs, Astron. J. 159, 60 (2020).

[91] E. F. Schlafly and D. P. Finkbeiner, Measuring reddening with Sloan Digital Sky Survey stellar spectra and recalibrating SFD, Astrophys. J. 737, 103 (2011).

[92] B. Moore, Constraints on the global mass-to-light ratios and on the extent of dark matter halos in globular clusters and dwarf spheroidals, Astrophys. J. Lett. 461, L13 (1996).

[93] R. Ibata, C. Nipoti, A. Sollima, M. Bellazzini, S. Chapman, and E. Dalessandro, Do globular clusters possess dark matter haloes? A case study in NGC 2419, Mon. Not. R. Astron. Soc. 428, 3648 (2013).

[94] P. J. E. Peebles, Dark matter and the origin of galaxies and globular star clusters, Astrophys. J. 277, 470 (1984).

[95] P. Creasey, L. V. Sales, E. W. Peng, and O. Sameie, Globular clusters formed within dark haloes I: presentday abundance, distribution, and kinematics, Mon. Not. R. Astron. Soc. 482, 219 (2019).

[96] B. F. Griffen, M. J. Drinkwater, P. A. Thomas, J. C. Helly, and K. A. Pimbblet, Globular cluster formation within the Aquarius simulation, Mon. Not. R. Astron. Soc. 405, 375 (2010).

[97] T. R. Saitoh, J. Koda, T. Okamoto, K. Wada, and A. Habe, Tidal disruption of dark matter halos around proto-globular clusters, Astrophys. J. 640, 22 (2006).

[98] L. Gao, S. D. M. White, A. Jenkins, F. Stoehr, and V. Springel, The subhalo populations of $\Lambda \mathrm{CDM}$ dark haloes, Mon. Not. R. Astron. Soc. 355, 819 (2004).

[99] J. F. Navarro, C.S. Frenk, and S.D. M. White, The structure of cold dark matter halos, Astrophys. J. 462, 563 (1996).
[100] D. Merritt, Evolution of the Dark Matter Distribution at the Galactic Center, Phys. Rev. Lett. 92, 201304 (2004).

[101] R. K. Leane, T. Linden, P. Mukhopadhyay, and N. Toro, Celestial-body focused dark matter annihilation throughout the galaxy, Phys. Rev. D 103, 075030 (2021).

[102] G. R. Blumenthal, S. M. Faber, R. Flores, and J. R. Primack, Contraction of dark matter galactic halos due to baryonic infall, Astrophys. J. 301, 27 (1986).

[103] O. Y. Gnedin and J. R. Primack, Dark Matter Profile in the Galactic Center, Phys. Rev. Lett. 93, 061302 (2004).

[104] A. M. Brown, T. Lacroix, S. Lloyd, C. Bœhm, and P. Chadwick, Understanding the $\gamma$-ray emission from the globular cluster 47 Tuc: evidence for dark matter? Phys. Rev. D 98, 041301 (2018).

[105] P. Amaro-Seoane, J. Casanellas, R. Schödel, E. Davidson, and J. Cuadra, Probing dark matter crests with white dwarfs and IMBHs, Mon. Not. R. Astron. Soc. 459, 695 (2016).

[106] C. Knigge, D. R. Zurek, M. M. Shara, and K. S. Long, A far-ultraviolet survey of 47 Tucanae. I. Imaging, Astrophys. J. 579, 752 (2002).

[107] R. L. Gilliland et al., A lack of planets in 47 Tucanae from a hubble space telescope search, Astrophys. J. Lett. 545, L47 (2000).

[108] T. Bringmann and M. Pospelov, Novel Direct Detection Constraints on Light Dark Matter, Phys. Rev. Lett. 122, 171801 (2019).

[109] I. Alkhatib et al. (SuperCDMS Collaboration), Light Dark Matter Search with a High-Resolution Athermal Phonon Detector Operated Above Ground, Phys. Rev. Lett. 127, 061801 (2021).

[110] M. A. Abramowicz, J. K. Becker, P. L. Biermann, A. Garzilli, F. Johansson, and L. Qian, No observational constraints from hypothetical collisions of hypothetical dark halo primordial black holes with galactic objects, Astrophys. J. 705, 659 (2009).

[111] M. Kesden and S. Hanasoge, Transient Solar Oscillations Driven by Primordial Black Holes, Phys. Rev. Lett. 107, 111101 (2011).

[112] P. W. Graham, R. Janish, V. Narayan, S. Rajendran, and P. Riggins, White dwarfs as dark matter detectors, Phys. Rev. D 98, 115027 (2018).

[113] J. Singh Sidhu and G. D. Starkman, Reconsidering astrophysical constraints on macroscopic dark matter, Phys. Rev. D 101, 083503 (2020).

[114] G. H. Jones, M. M. Knight, K. Battams, D. C. Boice, J. Brown, S. Giordano, J. Raymond, C. Snodgrass, J. K. Steckloff, P. Weissman et al., The science of sungrazers, sunskirters, and other near-sun comets, Space Sci. Rev. 214, 20 (2018).

[115] P. J. Francis, The demographics of long-period comets, Astrophys. J. 635, 1348 (2005).

[116] N. Brosch, V. Balabanov, and E. Behar, Small observatories for the UV, Astrophys. J. Suppl. Ser. 354, 205 (2014).

[117] J. Mathew, S. Ambily, A. Prakash, M. Sarpotdar, K. Nirmal, A. G. Sreejith, M. Safonova, J. Murthy, and N. Brosch, Wide-field ultraviolet imager for astronomical transient studies, Exp. Astron. 45, 201 (2018).

[118] S. Serjeant, M. Elvis, and G. Tinetti, The future of astronomy with small satellites, Nat. Astron. 4, 1031 (2020). 